\title{
Electric Quadrupolar Contributions in the Magnetic Phases of $\mathrm{UNi}_{4} \mathrm{~B}$
}

\author{
T. Yanagisawa $\odot,{ }^{1}$ H. Matsumori, ${ }^{1}$ H. Saito, ${ }^{1}$ H. Hidaka, ${ }^{1}$ H. Amitsuka, ${ }^{1}$ S. Nakamura, ${ }^{2}$ S. Awaji, ${ }^{2}$ \\ D. I. Gorbunov, ${ }^{3}$ S. Zherlitsyn, ${ }^{3}$ J. Wosnitza, ${ }^{3,4}$ K. Uhlî́rová, ${ }^{5}$ M. Vališka ${ }^{5},{ }^{5}$ and V. Sechovský $\odot^{5}$ \\ ${ }^{1}$ Department of Physics, Hokkaido University, Sapporo 060-0810, Japan \\ ${ }^{2}$ Institute for Materials Research, Tohoku University, Sendai 980-8577, Japan \\ ${ }^{3}$ Hochfeld-Magnetlabor Dresden (HLD-EMFL) and Würzburg-Dresden Cluster of Excellence ct.qmat, \\ Helmholtz-Zentrum Dresden-Rossendorf (HZDR), 01328 Dresden, Germany \\ ${ }^{4}$ Institut für Festkörper- und Materialphysik, TU Dresden, 01062 Dresden, Germany \\ ${ }^{5}$ Department of Condensed Matter Physics, Faculty of Mathematics and Physics, Charles University, 12116 Prague 2, Czech Republic
}

(Received 7 July 2020; revised 21 December 2020; accepted 2 March 2021; published 12 April 2021)

We present acoustic signatures of the electric quadrupolar degrees of freedom in the honeycomb-layer compound $\mathrm{UNi}_{4} \mathrm{~B}$. The transverse ultrasonic mode $C_{66}$ shows softening below $30 \mathrm{~K}$ both in the paramagnetic phase and antiferromagnetic phases down to $\sim 0.33 \mathrm{~K}$. Furthermore, we traced magnetic field-temperature phase diagrams up to $30 \mathrm{~T}$ and observed a highly anisotropic elastic response within the honeycomb layer. These observations strongly suggest that $\Gamma_{6}\left(E_{2 \mathrm{~g}}\right)$ electric quadrupolar degrees of freedom in localized $5 f^{2}(J=4)$ states are playing an important role in the magnetic toroidal dipole order and magnetic-field-induced phases of $\mathrm{UNi}_{4} \mathrm{~B}$, and evidence some of the $\mathrm{U}$ ions remain in the paramagnetic state even if the system undergoes magnetic toroidal ordering.

DOI: 10.1103/PhysRevLett.126.157201

The multipole formulation and its foundational concept in solid-state physics have been developed by intensive research on $f$-electron systems $[1,2]$. Recently, new theories based on the common language of "multipoles" [3,4] and "augmented multipoles" [5-9], which are spatially extended multipoles, have been evoked to construct a new framework for understanding various physical phenomena that are related to spin-orbit interactions beyond the differences in electron orbitals. In particular, the odd-parity augmented multipoles, including magnetic-electric and toroidal ones [10-12], have recently been extensively studied. Recent academic advances in understanding augmented multipoles have been preceded by theory rather than experiment. Therefore, it is necessary to demonstrate whether the new framework allows for a unified understanding of spontaneous spatial inversion symmetry breaking in metallic and insulating compounds. A major experimental challenge is to demonstrate odd-parity multipole ordering by observing cross-correlation phenomena and spontaneous spatial inversion-symmetry breaking in a suitable compound [5,8]. Among them we focus on the U-based honeycomb-layer compound $\mathrm{UNi}_{4} \mathrm{~B}$ [13-16], which is considered to be a good candidate for studying

Published by the American Physical Society under the terms of the Creative Commons Attribution 4.0 International license. Further distribution of this work must maintain attribution to the author(s) and the published article's title, journal citation, and DOI. augmented odd-parity multipoles, magnetic toroidal dipoles (MTDs), and the interplay with magnetoelectric phenomena $[12,17]$.

$\mathrm{UNi}_{4} \mathrm{~B}$ crystallizes in an orthorhombic structure (Space group; $\mathrm{Cmcm}, D_{2 h}^{17}$, No. 63) as shown in Fig. 1(a) [18]. Below $T_{N}=20.4 \mathrm{~K}$, this compound orders antiferromagnetically (AFM) in a magnetic structure where the magnetic moments are carried by two-thirds of the $\mathrm{U}$ ions $\left[\mathrm{U}_{\mathrm{AFM}}\right.$ sites in Fig. 1(b) form vortices in each pseudo-honeycomb plane], and one-third of the $\mathrm{U}$ ions $\left[\mathrm{U}_{\mathrm{PM} 1}\right.$ or $\mathrm{U}_{\mathrm{PM} 2}$ in Fig. 1(b)]

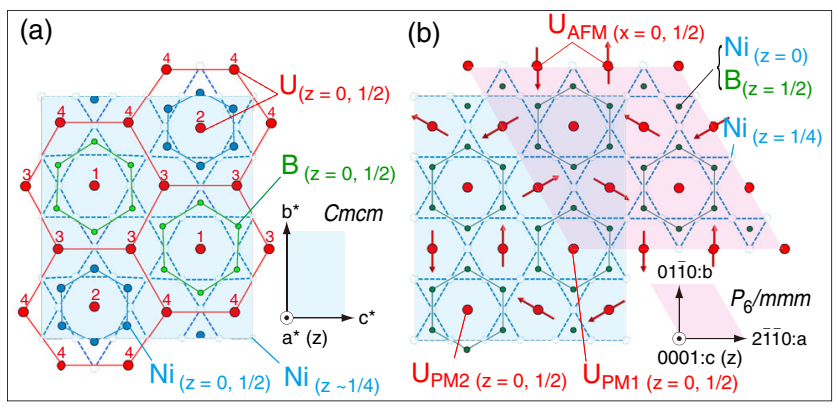

FIG. 1. Crystal and magnetic structure of $\mathrm{UNi}_{4} \mathrm{~B}$, reported for $B=0[16,18]$. (a) The pseudo-honeycomb network consists of two-thirds of $\mathrm{U}$ ions with $\mathrm{Cmcm}$ lattice. Red, blue, and green circles indicate $\mathrm{U}$ and $\mathrm{Ni}$ or $\mathrm{B}$ on the layer at $z=0,1 / 2$, respectively. Dashed lines with open circles show the (pseudo) Kagome layer of Ni atoms at $z \sim 1 / 4$. (b) Colored backgrounds with pink-rhomboidal and blue-rectangular shapes denote antiferromagnetic unit cells in hexagonal $(P 6 / \mathrm{mmm})$ and orthorhombic $(\mathrm{Cmcm})$ symmetry, respectively. 
remain in a paramagnetic $(\mathrm{PM})$ state. $[13,15,16]$ Assuming a hexagonal crystal structure $\left(P 6 / \mathrm{mmm}, D_{6 h}^{1}\right.$, No. 191), an exotic magnetic structure was proposed from neutron scattering experiments in earlier studies [16]. Since a slight deformation of the crystal structure from hexagonal to orthorhombic symmetry and different site occupations of $\mathrm{Ni}$ and $\mathrm{B}$ atoms have recently been reconfirmed by neutron and resonant $\mathrm{X}$-ray scattering studies as well as by ${ }^{11} \mathrm{~B}-\mathrm{NMR}$ measurements $[19,20]$, the previously proposed magnetic structure should be reconsidered based on the orthorhombic space group.

On the other hand, Hayami et al. has pointed out that such vortex-type magnetic structure in the (pseudo) honeycomb arrangement in $\mathrm{UNi}_{4} \mathrm{~B}$ can be understood in the framework of MTD order (see Fig. S1 in the Supplemental Material) $[12,21]$. Their theory has also predicted a new magnetoelectric effect: current-induced magnetization, which can occur in ferro-toroidal ordered metallic compounds, which has been experimentally confirmed in $\mathrm{UNi}_{4} \mathrm{~B}$ [17]. Recently, Yatsushiro and Hayami have reported on a theoretical investigation of an atomic scale MTD by taking into account orbital degrees of freedom with different parity [11]. This theory predicts that the orbital degrees of freedom in an interorbital space play an important role in stabilizing MTD order by odd-parity hybridization. However, the theory deals with the tetragonal point group $C_{4 \mathrm{v}}$, and the contribution of the orbital degrees of freedom such as even-parity electric multipolar moments to the noncollinear magnetic order in $\mathrm{UNi}_{4} \mathrm{~B}$ has not been investigated.

Another fascinating point and also an open question for this compound is a specific-heat anomaly at $T^{*} \sim 0.33 \mathrm{~K}$ of unknown origin [22]. Previous studies have explained that the narrowness of the $0.33 \mathrm{~K}$ anomaly in $\mathrm{UNi}_{4} \mathrm{~B}$ may be an indication of glassy behavior caused by the geometrical frustration of the paramagnetic $\mathrm{U}$ spins and their Kondo screening by the conduction electrons, since ac magnetic susceptibility and $\mu \mathrm{SR}$ measurements have shown no changes in the magnetic structure below $T^{*}$ [22]. Other possibilities, which have not been verified yet, are a nonmagnetic multipolar order of the PM-1/3 U ions and/or Schottky peak due to level splitting of the degenerate CEF ground state with low orthorhombicity. In order to study the electrical multipole contribution, it is useful to measure the elastic constants by ultrasound [23]. The elastic constants reflect the coupling of the strain field caused by the ultrasonic wave to the electric multipolar moments, which are described by orbital degrees of freedom of the CEF state. In this study, we show evidence of an electric multipolar ground state for $\mathrm{UNi}_{4} \mathrm{~B}$ based on ultrasound results. We further analyzed the possible contributions of electric quadrupoles to the noncollinear magnetic order and to the low-temperature specificheat anomaly (See Secs. (c)-(g) in the Supplemental Material [21] for experimental and analysis details).

Figure 2 shows the measured elastic constants of $\mathrm{UNi}_{4} \mathrm{~B}$ as a function of temperature. Here, the four ultrasonic

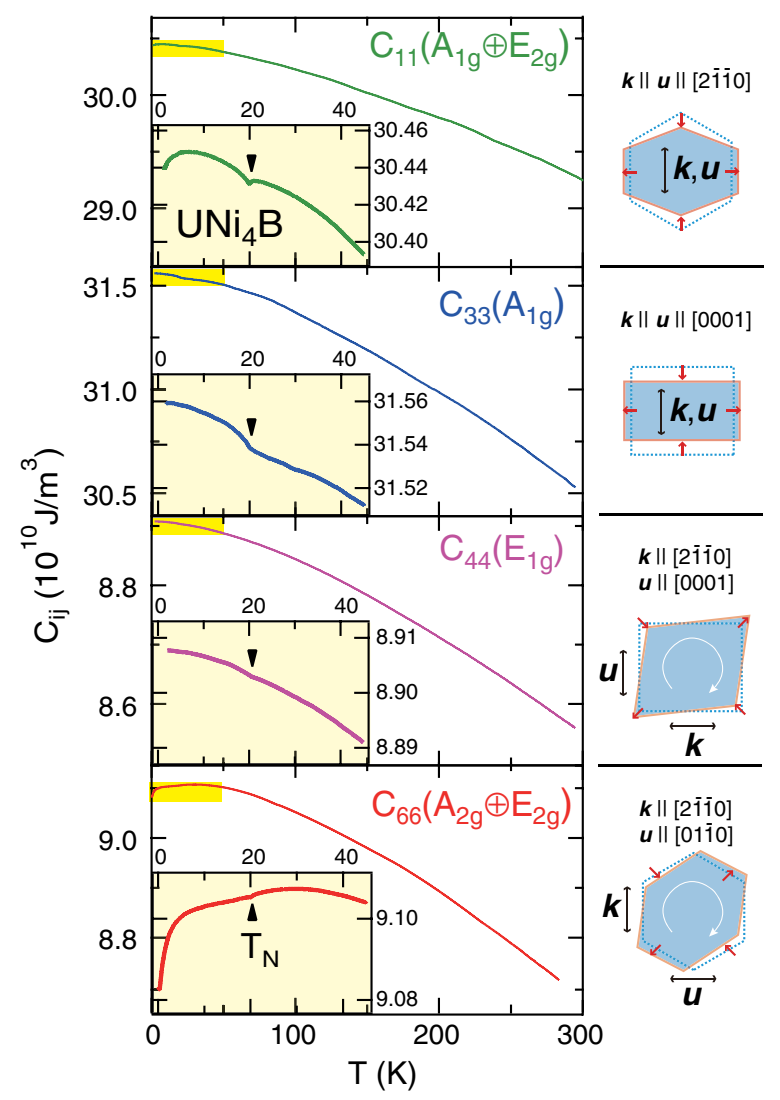

FIG. 2. Elastic constants of $\mathrm{UNi}_{4} \mathrm{~B}$ as a function of temperature. The insets in each panel show enlarged views of the data below $50 \mathrm{~K}$. Illustrations of the distorted hexagon and/or rectangle indicate the lattice strain, which is induced by the respective ultrasonic mode (See Table SI in the Supplemental Material) $[21,25,26]$.

modes are symmetrized using the hexagonal point group $D_{6 h}$. The ultrasound induces local strain and rotation fields [21] in the solid sample as shown by the schematic illustrations in each panel of Fig. 2. The local strain and rotation field behave as conjugate fields for electric quadrupole or electric hexadecapole moments [24]. Responses of the multipoles can be observed as sound-velocity change and ultrasonic attenuation via electron-phonon interaction. By comparing the temperature dependence of the four ultrasonic modes, it becomes obvious that only the transverse ultrasonic mode $C_{66}$ exhibits a softening below $30 \mathrm{~K}$ in the PM phase with a kink at $T_{N}$ and keeps decreasing in the antiferromagnetic (AFM) phase down to $\sim 0.33 \mathrm{~K}$ [Fig. 3(b)].

The other ultrasonic modes, the longitudinal $C_{11}, C_{33}$, and transverse $C_{44}$ modes, do not show such softening particularly below $T_{\mathrm{N}}$. From the selection rules within the category of even-parity multipoles, the results indicate that an electric quadrupole with $\Gamma_{6}\left(\mathrm{E}_{2 \mathrm{~g}}\right)$ symmetry (in the hexagonal point group) is active in $\mathrm{UNi}_{4} \mathrm{~B}$. In the first stage of the analysis, we consider the conventional localized $5 f$-electronic states with even-parity CEF levels and 

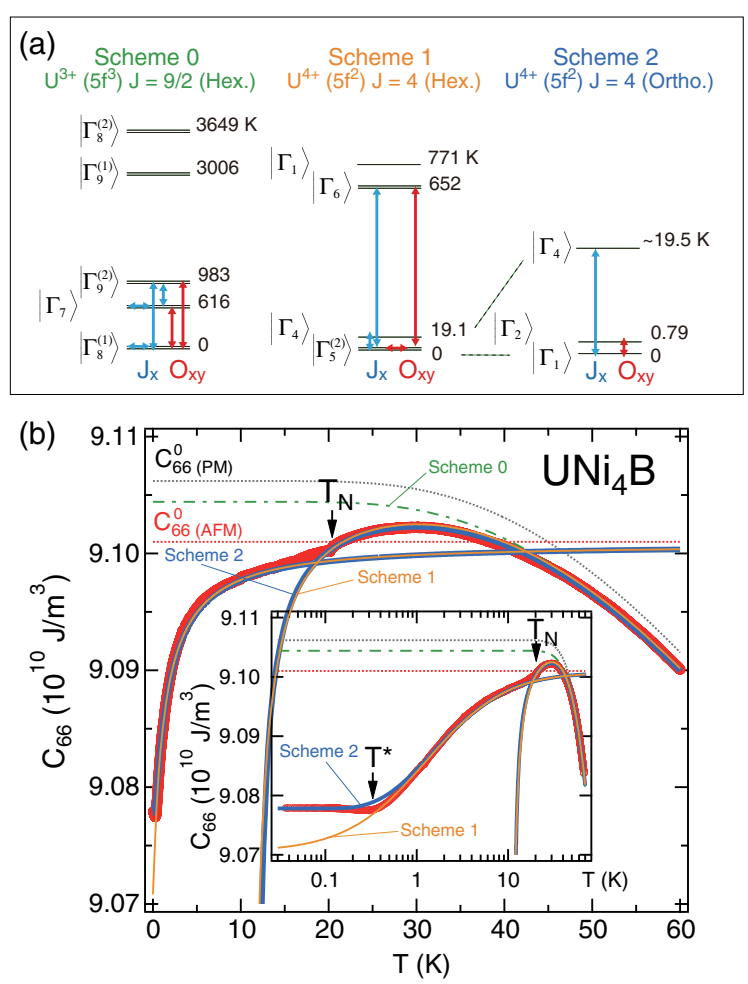

FIG. 3. (a) CEF level schemes used for the present analysis (see Table SIII, SIV, and SV in the Supplemental Material [21]). Blue and red arrows indicate selection rules where finite matrix elements exist for magnetic dipole $J_{x}$ and electric quadrupole $O_{x y}$, respectively. (b) Temperature dependence of the elastic constant $C_{66}$, compared with calculations based on the quadrupolar susceptibility using the CEF level schemes shown in panel (a): Scheme 0 (green), scheme 1 (orange), and scheme 2 (blue) as shown by dashed and solid curves. The dotted black and red curves show the background of the elastic constant $C_{66}^{0}$ in the PM and AFM phases, respectively. The inset shows an enlarged view of the low-temperature region on a $\log -T$ scale.

multipoles for the analysis of the elastic responses. Here, we do not take into account the contribution from oddparity multipoles, because such effect only couples to the elastic strain through cross-correlation with the application of appropriate external fields with odd-parity space-inversion symmetry $[6,8]$. As shown below, the current analysis mimics the experimental results well that the odd-parity mixing inherent in the CEF is negligible. The softening in $C_{66}$ in the paramagnetic phase could, however, not be reproduced by the previously proposed CEF level scheme [scheme 0 in Fig. 3(a)] [27] with the localized $5 f$-electronic state of $\mathrm{U}^{3+}$ having a hexagonal symmetry because the $5 f^{3}$ $(J=9 / 2)$ state only shows $\Gamma_{6}$-quadrupolar excitation $\left[O_{x y}=\sqrt{3}\left(J_{x} J_{y}+J_{y} J_{x}\right) / 2\right]$ in the off-diagonal elements between the ground-state Kramers doublet and the excited levels that are separated by an energy gap of over $600 \mathrm{~K}$, as shown in Fig. 3(a) [for matrix elements, see Sec. (f) in the Supplemental Material [21]].
In order to reproduce the softening in the $C_{66}$ mode at higher temperature than $T_{N}$, we propose a different CEF model [scheme 1 in Fig. 3(a)] with a localized $5 f^{2}(J=4)$ state of $\mathrm{U}^{4+}$, which has a pseudo-triplet ground state. The CEF parameters of CEF scheme 1 (Table SIV in the Supplemental Material [21]) are set to reproduce simultaneously the elastic softening in $C_{66}$, no elastic softening in $C_{44}$, and also the magnetic susceptibility below $50 \mathrm{~K}$ at the same time (for details see Fig. S3 in the Supplemental Material [21]). The coupling constant $g_{\Gamma}^{\prime}$ of quadrupolar inter-site interactions is described by the Hamiltonian $\mathcal{H}_{\mathrm{MM}}=-\sum_{\alpha} g_{\Gamma_{6}}^{\prime}\left\langle O_{x y}\right\rangle O_{x y}^{\alpha}$ for sublattices $\alpha$ and a $\Gamma_{6}$ symmetry quadrupolar moment $O_{x y}$ [see Eqs. (17)-(21) in the Supplemental Material) [21,23,28]. Our analysis reveals a positive value $g_{\Gamma 6(\mathrm{PM})}^{\prime}=+0.42 \mathrm{~K}$, which strongly suggests the presence of a weak but finite ferro-type quadrupolar interaction in the $\mathrm{PM}$ phase of $\mathrm{UNi}_{4} \mathrm{~B}$. A possible relationship between the ferrotype MTD order of this system and the ferro-quadrupolar interaction is nontrivial and remains an open question. The softening of $C_{66}$ in the AFM phase can also be analyzed using the same CEF parameters, since the $\mathrm{PM}-1 / 3 \mathrm{U}$ ions (on the $\mathrm{U}_{\mathrm{PM} 1}$ or $\mathrm{U}_{\mathrm{PM} 2}$ sites) located in the center of the pseudo-honeycomb plane are not affected by the on-site electric or magnetic fields formed by the MTD moment. Although the global inversion symmetry on the $\mathrm{U}_{\mathrm{PM} 1}$ and $\mathrm{U}_{\mathrm{PM} 2}$ sites is broken due to the MTD order, the odd-parity CEF states and multipole will, however, not be active on the PM-1/3 U sites when only considering the $J=4$ Hilbert space and assuming weak orbital coupling between the $\mathrm{U}$ and $\mathrm{Ni}$ or B ions.

The inset in Fig. 3(b) shows the temperature dependence of $C_{66}$ on a $\log -T$ scale. Calculations of the quadrupolar susceptibility of the PM-1/3 U ions using the CEF scheme 1 (orange curve) [21] with hexagonal symmetry well reproduces the softening in the AFM phase down to $\sim 0.33 \mathrm{~K}$. Here, the contribution from the AFM ordered$2 / 3 \mathrm{U}$ ions is assumed as constant background and the CEF level scheme of the PM-1/3 U ions is not changed from the PM phase. Note that the intersite quadrupolar interaction for the AFM phase obtained by our analysis is negative with $g_{\Gamma 6(\mathrm{AFM})}^{\prime}=-0.045 \mathrm{~K}$, which means the presence of antiferro-quadrupolar (AFQ) interaction in the AFM phase. In order to reproduce the temperature dependence down to $T^{*}$ in the present analysis, it is unlikely that $g_{\Gamma 6(\mathrm{AFM})}^{\prime}$ takes a positive value. Therefore, we can conclude that in the ordered phase there is an antiferro-quadrupolar interaction between the $\mathrm{U}_{\mathrm{PM}}$ 's [in Fig. 1(b)], which is fundamentally the opposite to that for $\mathrm{U}_{\mathrm{AFM}}$ in the PM phase.

The calculated result using scheme 1 deviates from the experimental data below $T^{*} \sim 0.33 \mathrm{~K}$. This deviation indicates a small CEF splitting of the ground-state doublet. Such splitting might occur for two reasons: (i) symmetry lowering due to ordering of the remaining $\mathrm{PM}-1 / 3 \mathrm{U}$ multipolar moments or (ii) the crystal structure essentially 

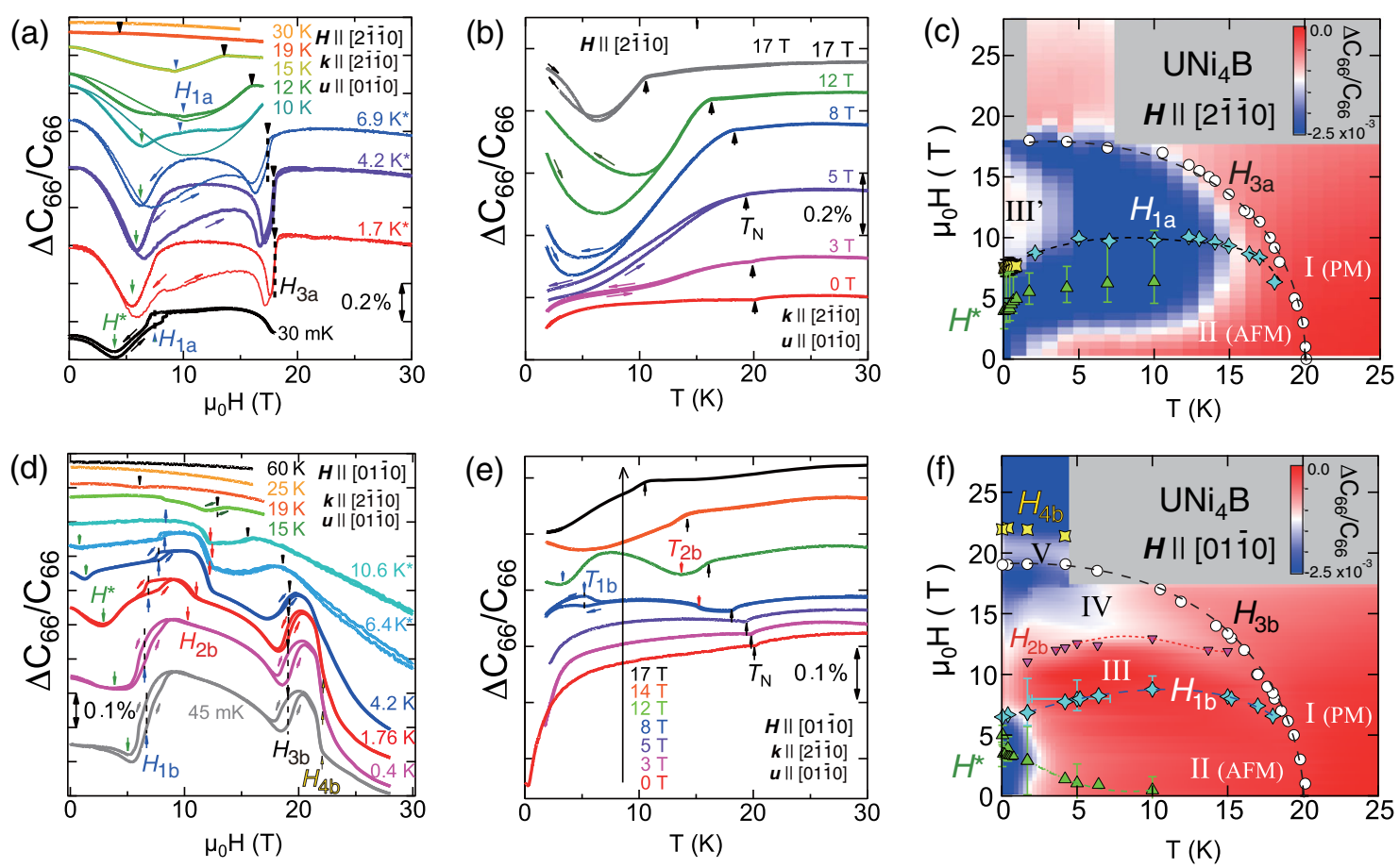

FIG. 4. Magnetic-field dependence of $C_{66}$ for (a) $H \|[2 \overline{1} \overline{1} 0]$ and (d) $H \|[01 \overline{1} 0]$ of $\mathrm{UNi}_{4} \mathrm{~B}$ at selected temperatures. $C_{66}$ vs temperature under various magnetic fields for (b) $H \|\left[\begin{array}{lll}2 & 1 & 0\end{array}\right]$ and (e) $H \|[01 \overline{1} 0]$. In each panel, vertical arrows indicate elastic anomalies, which correspond to phase boundaries in the magnetic field-temperature phase diagram of $\mathrm{UNi}_{4} \mathrm{~B}$ for (c) $H \|[2 \overline{1} \overline{1} 0]$ and (f) $H \|[01 \overline{1} 0]$. Dotted and dashed lines are guides for the eyes. The relative change of the elastic constant $C_{66}$ is highlighted by the color map from zero (red) to large negative (blue) values.

having lower symmetry. Since it has been confirmed that the point group of the $\mathrm{U}$ ions in the present system has orthorhombic symmetry, we have modified the CEF scheme 1 by adding low orthorhombicity [as CEF parameter $B_{2}^{2}$ and $B_{4}^{2}$ for Steven's operator $O_{2}^{2}$ and $O_{4}^{2}$ [see Eqs. (3)-(11) in the Supplemental Material [21] ] [3,21,29,30], respectively, have finite values] to split the ground-state non-Kramers doublet with a gap of $\Delta \sim 0.79 \mathrm{~K}$. This CEF scheme 2 [Fig. 3(a), Table SV in the Supplemental Material [21] ] reproduces the leveling off of $C_{66}$ (blue curve) as well as a Schottky-type specific-heat peak at $\Delta / 2.398 \sim 0.33 \mathrm{~K}$ [22]. We, therefore, conclude that the mentioned $T^{*}$ transition, found in specific heat, is a Schottky anomaly due to the orthorhombicity of the crystal. It should be noted that we determined the magneticfield dependence of $T^{*}$ and its anisotropy for $H \|\left[\begin{array}{lll}2 & \overline{1} & 1\end{array}\right]$ and [0110] in the elastic constant $C_{66}$. Our results are roughly consistent with those of earlier studies [16] (see Fig. S8 in the Supplemental Material [21]).

The temperature and magnetic-field dependence of the elastic constant $C_{66}$ are shown in Figs. 4(a) and 4(b) for $H \|[2 \overline{1} \overline{1} 0]$ and in Figs. 4(d) and 4(e) for $H \|[01 \overline{1} 0]$. In Fig. 4(a), the data with an asterisk are obtained in pulsedmagnetic-field measurements up to $60 \mathrm{~T}$ (see Fig. S9 in the Supplemental Material [21]). We obtained $C_{66}$ vs $H \|[01 \overline{1} 0]$ up to 28 T, shown in Fig. 4(d), in static magnetic fields using the cryogen-free hybrid magnet system equipped with a dilution refrigerator [31]. Several elastic anomalies are observed, which are indicated by arrows; $H^{*}, H_{1 \mathrm{~b}}$, and $H_{3 \mathrm{~b}}$ for $H \|[2 \overline{1} \overline{1} 0], H^{*}, H_{1 \mathrm{a}}, H_{2 \mathrm{a}}, H_{3 \mathrm{a}}$, and $H_{4 \mathrm{a}}$ for $H \|[01 \overline{1} 0]$. The data display both up and down sweeps of the magnetic field. We observe hysteretic regions below $H_{3 \mathrm{a}}$. The elastic responses in $C_{66}$ show a large in-plane (0001) anisotropy for $H \|[2 \overline{1} \overline{1} 0]$ and $H \|[01 \overline{1} 0]$, while the magnetization does not show such strong anisotropies [16]. The positions of the elastic anomalies are indicated as well in the magnetic field-temperature $(H-T)$ phase diagrams as shown in Figs. 4(c) and 4(f). Here, a number of phases are distinguishable for $H \|[01 \overline{1} 0]$; PM phase I, AFM phase II, the spin-reoriented AFM phase III, the spin-flop phase IV with hysteresis, which was previously evidenced by magnetization data [16], and a newly found unknown phase V. The obtained phase boundaries are consistent with the previously reported phase diagram [16] except for the high-magnetic-field region. On the other hand, the $H-T$ phase diagram for $H \|[2 \overline{1} \overline{1} 0]$ is quite different with less phases and completely different elastic responses. The background red-white-blue color code in Figs. 4(c) and 4(f) represents the relative changes in $C_{66}$ from larger to smaller stiffness. Remarkably, the contour plot shows a significant difference in stiffness for $H \|[01 \overline{1} 0]$ and [2 $\overline{1} \overline{1} 0]$, though no difference was detected in magnetization. This new observation clearly indicates a possible contribution of electric quadrupoles of the PM-1/3 U ions, which modifies the spin-reorientation process as well. 
In the low-magnetic field and low-temperature regions for both $H \|[01 \overline{1} 0]$ and $H \|[2 \overline{1} \overline{1} 0]$, the blue color indicates enhanced contributions from the $\Gamma_{6}\left(E_{2 \mathrm{~g}}\right)$-electric quadrupoles, i.e., this system has incoherent fluctuations of the electric quadrupoles due to the CEF pseudo-doublet ground state. In general, the ground-state doublet (including nonKramers doublet) splits due to the Zeeman effect (with mixing of excited-level wave functions) in an external magnetic field or in the internal fields produced by magnetic order, and the quadrupole degrees of freedom become inactive, resulting in a hardening of $C_{66}$. Remarkably, $C_{66}$ experiences a softening in phase IV for $H \|[01 \overline{1} 0]$ and in the intermediate temperature range of phase III' for $H \|\left[\begin{array}{lll}2 & \overline{1} & 0\end{array}\right]$ compared with the changes in the other phases. This fact suggests a reactivation of the quadrupole degrees of freedom with $\Gamma_{6}\left(E_{2 \mathrm{~g}}\right)$ symmetry above $\sim 12 \mathrm{~T}$ in the low-temperature region for $H \|[01 \overline{1} 0]$.

In summary, we conclude that the electric-quadrupole degrees of freedom play a crucial role in the low-temperature properties of $\mathrm{UNi}_{4} \mathrm{~B}$, leading to anisotropic $H-T$ phase diagrams and a newly revealed field-induced phase V. The observed softening of the $C_{66}$ elastic constant can be well explained by quadrupolar-strain interactions. The corresponding CEF analysis results in a new level scheme (scheme 2) taking into account the established orthorhombic symmetry and the $5 f^{2}(J=4)$ state of uranium ions. Furthermore, in this level scheme the puzzling specific-heat anomaly at $\sim 0.33 \mathrm{~K}$ can be understood as a Schottky anomaly due to a small level splitting of the non-Kramers ground-state doublet. Moreover, our results confirm that some of the U ions stay disordered in the MTD ordered phase. Further theoretical considerations would be of interest to clarify the quadrupolar contributions in the toroidal order and anisotropic elastic response in the newly established magnetic-field-temperature phases.

We thank C. Tabata, S. Hayami, and H. Kusunose for fruitful discussions. The present research was supported by JSPS KAKENHI Grants No. JP17K05525, No. JP15KK0146, No. JP15K13509, No. JP15H05885, No. JP15K21732, and the Strategic Young Researcher Overseas Visits Program for Accelerating Brain Circulation from JSPS. This study was partly supported by Hokkaido University, Global Facility Center (GFC), Advanced Physical Property Open Unit (APPOU), funded by MEXT under Support Program for Implementation of New Equipment Sharing System Grant No. JPMXS0420100318. A part of this work was performed at the High Field Laboratory for Superconducting Materials, Institute for Materials Research, Tohoku University (Project No. 17H0063). We also acknowledge the support of the Hochfeld-Magnetlabor Dresden at HZDR, a member of the European Magnetic Field Laboratory (EMFL), and the Deutsche Forschungsgemeinschaft (DFG) through SFB 1143 (Project No. 247310070) and the Würzburg-Dresden
Cluster of Excellence on Complexity and Topology in Quantum Matter-ct.qmat (EXC 2147, Project No. 390858490). The sample used in HZDR has been grown and characterized in the Materials Growth and Measurement Laboratory MGML which is supported within the program of Czech Research Infrastructures (Project No. LM2018096).

[1] P. Santini, S. Carretta, G. Amoretti, R. Caciuffo, N. Magnani, and G.H. Lander, Multipolar interactions in f-electron systems: The paradigm of actinide dioxides, Rev. Mod. Phys. 81, 807 (2009).

[2] Y. Kuramoto, H. Kusunose, and A. Kiss, Multipole orders and fluctuations in strongly correlated electron systems, J. Phys. Soc. Jpn. 78, 072001 (2009).

[3] H. Kusunose, Description of multipole in f-electron systems, J. Phys. Soc. Jpn. 77, 064710 (2008).

[4] Y. Kuramoto, Electronic higher multipoles in solids, Prog. Theor. Phys. Suppl. 176, 77 (2008).

[5] S. Hayami, Y. Yanagi, and H. Kusunose, Bottom-up design of spin-split and reshaped electronic band structures in antiferromagnets without spin-orbit coupling: Procedure on the basis of augmented multipoles, Phys. Rev. B 102, 144441 (2020).

[6] S. Hayami, M. Yatsushiro, Y. Yanagi, and H. Kusunose, Classification of atomic-scale multipoles under crystallographic point groups and application to linear response tensors, Phys. Rev. B 98, 165110 (2018).

[7] S. Hayami and H. Kusunose, Microscopic description of electric and magnetic toroidal multipoles in hybrid orbitals, J. Phys. Soc. Jpn. 87, 033709 (2018).

[8] H. Watanabe and Y. Yanase, Group-theoretical classification of multipole order: Emergent responses and candidate materials, Phys. Rev. B 98, 245129 (2018).

[9] M.-T. Suzuki, H. Ikeda, and P. M. Oppeneer, First-principles theory of magnetic multipoles in condensed matter systems, J. Phys. Soc. Jpn. 87, 041008 (2018).

[10] M. Yatsushiro and S. Hayami, Odd-parity multipoles by staggered magnetic dipole and electric quadrupole orderings in CeCoSi, J. Phys. Soc. Jpn. 89, 013703 (2020).

[11] M. Yatsushiro and S. Hayami, Atomic-scale magnetic toroidal dipole under odd-parity hybridization, J. Phys. Soc. Jpn. 88, 054708 (2019).

[12] S. Hayami, H. Kusunose, and Y. Motome, Toroidal order in metals without local inversion symmetry, Phys. Rev. B 90, 024432 (2014).

[13] J. A. Mydosh, G. J. Nieuwenhuys, S. A. M. Mentink, and A. A. Menovsky, Unusual magnetic ordering in the intermetallic compound $\mathrm{UNi}_{4} \mathrm{~B}$, Philos. Mag. B 65, 1343 (1992).

[14] S. A. M. Mentink, H. Nakotte, A. de Visser, A. A. Menovsky, G. J. Nieuwenhuys, and J.A. Mydosh, Reduced-moment antiferromagnetism in single-crystal $\mathrm{UNi}_{4} \mathrm{~B}$, Physica (Amsterdam) 186-188B, 270 (1993).

[15] S. A. M. Mentink, A. Drost, G. J. Nieuwenhuys, E. Frikkee, A. A. Menovsky, and J. A. Mydosh, Magnetic Ordering and Frustration in Hexagonal $\mathrm{UNi}_{4} \mathrm{~B}$, Phys. Rev. Lett. 73, 1031 (1994). 
[16] S. A. M. Mentink, G. J. Nieuwenhuys, H. Nakotte, A. A. Menovsky, A. Drost, E. Frikkee, and J. A. Mydosh, Magnetization and resistivity of $\mathrm{UNi}_{4} \mathrm{~B}$ in high magnetic fields, Phys. Rev. B 51, 11567 (1995).

[17] H. Saito, K. Uenishi, N. Miura, C. Tabata, H. Hidaka, T. Yanagisawa, and H. Amitsuka, Evidence of a New CurrentInduced Magnetoelectric Effect in a Toroidal Magnetic Ordered State of $\mathrm{UNi}_{4} \mathrm{~B}$, J. Phys. Soc. Jpn. 87, 033702 (2018).

[18] Y. Haga, A. Oyamada, T. D. Matsuda, S. Ikeda, and Y. Onuki, Crystal structure of frustrated antiferromagnet $\mathrm{UNi}_{4}$ B, Physica (Amsterdam) 403B, 900 (2008).

[19] C. Tabata (to be published).

[20] R. Takeuchi, Y. Kishimoto, H. Kotegawa, H. Harima, Y. Homma, F. Honda, A. Nakamura, Y. Shimizu, D. X. Li, D. Aoki, and $\mathrm{H}$. Tou, ${ }^{11} \mathrm{~B}-\mathrm{NMR}$ investigation for crystal structure in antiferromagnet $\mathrm{UNi}_{4} \mathrm{~B}$, J. Phys. Soc. Jpn. Conf. Proc. 29, 013001 (2020).

[21] See Supplemental Material at http://link.aps.org/ supplemental/10.1103/PhysRevLett.126.157201 for (a) schematic illustration of the magnetic toroidal order, (b) indexing a hexagonal system with four axes, (c) experimental details, (d) formulation of multipolar susceptibility, (e) definition of multipolar moments and equivalent operator expressions, (f) matrix elements of magnetic dipole and electric quadrupoles, $(\mathrm{g})$ consistency test for CEF calculations of the magnetic susceptibility, (h) elastic constant $C_{66}$ and calculated quadrupolar susceptibility at low temperatures ( $<1 \mathrm{~K}$ ), (i) anisotropy of the $H-T$ phase diagram and the $330 \mathrm{mK}$ anomaly, and (j) elastic response of the $C_{66}$ mode under pulsed magnetic fields above $30 \mathrm{~T}$.
[22] R. Movshovich, M. Jaime, S. Mentink, A. A. Menovsky, and J. Mydosh, Second Low-Temperature Phase Transition in Frustrated $\mathrm{UNi}_{4}$ B, Phys. Rev. Lett. 83, 2065 (1999).

[23] B. Lüthi, in , Physical Acoustics in the Solid State (Springer, Berlin, 2006).

[24] R. Kurihara, K. Mitsumoto, M. Akatsu, Y. Nemoto, T. Goto, Y. Kobayashi, and M. Sato, Critical slowing down of quadrupole and hexadecapole orderings in iron pnictide superconductor, J. Phys. Soc. Jpn. 86, 064706 (2017).

[25] V. Dohm and P. Fulde, Effective spin models for spin-phonon chains by flow equations, Z. Phys. B 21, 369 (1975).

[26] T. Goto, A. Tamaki, T. Fujimura, and H. Unoki, Quadrupolar Response and Rotational Invariance of Singlet Ground State System; $\mathrm{HoVO}_{4}$, J. Phys. Soc. Jpn. 55, 1613 (1986).

[27] A. Oyamada, M. Kondo, T. Itou, S. Maegawa, D. X. Li, and Y. Haga, Spin dynamics in a triangular antiferromagnet $\mathrm{UNi}_{4}$ B, J. Phys. Conf. Ser. 145, 012044 (2009).

[28] P. Thalmeier and B. Lüthi, in Handbook on the Physics and Chemistry of Rare Earths, edited by J. K. A. Gschneider and L. Eyring (North-Holland, Amsterdam, 1991), Vol. 14, p. 311.

[29] K. W. H. Stevens, Matrix elements and operator equivalents connected with the magnetic properties of rare earth ions, Proc. Phys. Soc. London Sect. A 65, 209 (1952).

[30] M. Hutchings, Point-Charge Calculations of Energy Levels of Magnetic Ions in Crystalline Electric Fields(Academic Press, New York, 1964), pp. 227-273.

[31] K. Watanabe, G. Nishijima, S. Awaji, K. Takahashi, K. Koyama, N. Kobayashi, M. Ishizuka, T. Itou, T. Tsurudome, and J. Sakuraba, Performance of a cryogen-free 30 T-class hybrid magnet, IEEE Trans. Appl. Supercond. 16, 934 (2006). 